more flurried from observing the fresh hæmorrhage, he passes up his hand, and feels nothing but the uterus tightly contracted around the placenta. Mistaking this for the latter, he gets his fingers into the hollow of the sacrum behind it, with the object of scooping out the after-birth, which he supposes to be (what the old nurses call) " grown to the side". At last, by means of his nails, he succeeds in separating the posterior wall of the vagina from its attachment to the uterus, and gets his hand into the pouch of Douglas, and of course, into the peritoneal cavity. Still unaware of his mistake, he grasps the fundus uteri from behind ; and, thus obtaining a good purchase, he tears away the entire organ; and then at last, with the appearance of the intestines, the horrible conviction of his blunder dawns upon him.

Such is the manner in which we may reasonably suppose that many of these awful cases of malapraxis take place. I need scarcely remark that such accidents ought to be impossible to any one possessed of the smallest amount of skill and anatomical knowledge.

To pass on, however, to the manipulation necessary in these cases : if the cord be tightly encircled by the os uteri, the constriction should be overcome by insinuating the tips of the fingers into the os in a conical form; whilst the right hand all this time is making counterpressure upon the fundus uteri, so as to steady that organ. Should these precautions be neglected, the connections between the vagina and the uterus may be put very injuriously on the stretch, especially if the circular fibres of the os oppose much resistance to the introduction of the hand. As the tips of the fingers pass through the os, they should be gradually expanded and separated from one another, until, by sheer fatigue, they overcome the contraction of the uterine fibres, so as to allow the passage of the entire hand into the uterus. When this is accomplished, the next step is to pass up the hand sufficiently high to reach the placenta. The distance which it has to pass before this can be felt will depend very much upon the position of the placenta and the degree of contraction of the uterus. If the placenta be attached, as it usually is, to the fundus uteri, or if the uterus be in a flaccid condition, it will be necessary to pass the hand much further than when the placenta is attached lower down, or when the uterus is well contracted. I have sometimes had to pass the hand quite into the epigastric region in search of a retained placenta. As soon as the placenta is arrived at, the fingers should be spread out, taking care not to entangle them in the membranes, until the circumference of the placenta can be felt. If any portion of the circumference be already detached, the tips of the fingers should be cautiously inserted between this portion and the inner surface of the uterus, and the placenta gradually peeled off. All this time, the right hand, externally applied, steadies the portion of the uterus from which the left hand is detaching the placenta, and enables the accoucheur to estimate the exact thickness of the uterine walls included between the hands, so that he can avoid digging his nails into the substance of the uterus. There is sometimes considerable danger of such an accident when the adhesions are very firm and close. There is also considerable danger of leaving portions of placenta behind : a risk that one can readily comprehend in such cases as those described by Dr. Ramsbotham, who states: "I have opened more than one body where a part was left adherent to the uterus, and where, on making a longitudinal section of the organs, and examining the cut edges, I could not determine the boundary line between the uterus and the placenta, so intimate an union had taken place between them." In all such difficult cases, it will be necessary to sever the adhesions by using the finger-nails with a kind of sawing motion from side to side. The tips of the fingers are placed in a line like the edge of a saw, keeping the palm towards the placenta and the knuckles towards the uterus, and the sawing motion is continued very slowly and gradually, until the entire placenta is separated and falls into the hollow of the hand. This proceeding sometimes requires a great deal of patience, and is exceedingly tiring; but the accoucheur should take his time about it, working with both hands, and making his ground sure as he goes on, and not withdrawing his hand with the placenta until he is certain that he has brought away every part of it that can be safely separated. It is very seldom, comparatively, that the adhesions are so firm that this cannot be done. Should this, however, be the case, we have a choice of evils : either to run the risk of causing secondary hæmorrhage and septicæmia by leaving portions behind, or of causing metritis from injury to the uterus in bringing them away. For my own part, I think that the last of these two courses is the least dangerous, except in very unusual cases. I have notes of only two instances in which it was necessary to leave any portion of consequence behind. Fortunately, in both, the pieces were expelled on the third day, without having caused any untoward symptoms, although in one the piece expelled was as large as a hen's egg. Of course, in all such instances, the dangers of septicæmia should be guarded against as much as possible by the frequent use of vaginal injections containing Condy's or other disinfectant Huids.

\section{A REPORT ON INFECTION.}

\author{
By JOHN HADDON, M.A., M.D., Eccles, Manchester.
}

IN I872, at the annual meeting of the Lancashire and Cheshire Branch of the British Medical Association, on the suggestion of Dr. Ransome, a committee was formed to inquire into the subject of infection, with a view to rendering our knowledge, if possible, more exact.

The committee, to this end, drew up a letter, asking assistance in obtaining facts; and with this letter a form, containing such questions as we wished answered, was sent to a large number of the profession in Great Britain and Ireland.

The answers received up to this date are not so numerous as could have been desired; nevertheless, they contain some important facts, and, at the request of the committee, I have drawn up the following short report for publication in the JOURNAL, hoping that it may direct attention to the subject, and induce the whole body of members to assist in the undertaking.

By " infectious" is generally understood the power of communicating disease by some emanations from the body of the individual affected. The "infection" is the something which affects the healthy body and produces in it disease. Of the real essence of infections very little is known; but there can be little doubt that in a few years our knowledge in this direction will be materially advanced; and we may yet hope to be able to separate, collect, and bottle up the various infective agents, which are so prolific in our large towns, and so deadly in their action on the human body. For such progress, we look to physiologists, who have the wards of a hospital as a field of observation, and the laboratory, with all modern means of experimental research, in which to unravel the physiology of disease. There are, however, many facts connected with the natural history of infection which can be best observed by the general practitioner, more especially in thinly populated country districts; and it is through the busy practitioner, and not through the experimental physiologist, that the aim of the committee is to be attained.

The diseases selected for investigation are small-pox, measles, scarlet fever, diphtheria, typhus, typhoid, whooping-cough, and mumps. These ailments are seldom entirely absent from a town of any size in this country, and the general practitioner is frequently asked questions about infection with regard to them, which he has to answer in a way that reflects little credit on the medical profession, and makes one wonder that no investigation has been undertaken by the profession as a body, with the view of rendering our knowledge more definite, and the information very naturally required of us more precise and reliable. We have a few examples of an energetic and observant physician investigating the incubation period of scme particular disease or class of diseases; but, so far as I am aware, the Lancashire and Cheshire Branch of the British Medical Association affords the first example of a large number of members of the medical profession agreeing to collect facts which may help towards the attainment of more exact knowledge regarding infection ; and, since the subject is of such importance to mankind over the whole world, it is to be hoped that its efforts will be appreciated, and the work helped on by the profession abroad as well as at home.

Text-books on medicine teach that a healthy individual associating with a person suffering from any infectious disease is liable to receive the infection into his system through some channel; that, after a certain period known as the " period of incubation", during which time it is supposed that the infection in the blood has been undergoing a process somewhat similar to fermentation, it is rendered able to affect the equilibrium of health. The infected individual then feels what are called the "premonitory" symptoms; and, after a certain time, varying with the particular disease, the sign or symptom which determines the species is developed.

The "period of incubation" then first attracts attention, and what we have to determine is its duration. Now, the text-books do not fail to state the duration of incubation in each of the diseases selected; but in every one it is indefinite, so that it is stated as varying from so many to so many days. Thus, in small-pox, it is from twelve to fourteen days ; in measles, ten to sixteen days ; in diphtheria, three to twenty-two days ; in typhus, a few minutes to twenty-one days ; in typhoid, ditto ; in whooping-cough, five to six days ; in mumps, eight to twenty-two days. That may be accepted as the present teaching with regard to the incubation in each of these diseases, and no one will deny that it is far from definite or satisfactory. What the committee desires is, if possible, to fix the precise duration, since, from all we know, under the 
same conditions, its duration will be the same. Thus, if two healthy persons enter the sick room of a scarlet fever patient, keep together, and remain for some time breathing the atmosphere of the room, one would expect that, if they both caught the infection, they would become ill at the same time ; but, if a third person be in their company, and handle the patient or anything he uses, so as to bring some of the secretions of the patient into contact with a sore, or into his mouth, we need not be astonished if, the latter being infected, the incubation period should be different.

The next point which calls for notice is the "duration of infection", and this divides itself into two parts: $\mathbf{I}$. When the disease is first infectious; and 2. How long it continues infectious.

It has been the custom to isolate cases when the pathognomonic sign was discovered; but, though it may appear to some unimportant, it now seems probable that some diseases become infectious so soon as the equilibrium of health is upset. Dr. W. Squire, in a paper on the Period of Infection in Epidemic Disease, which is a valuable contribution to the literature of the subject, lays stress upon the fact that measles and whooping-cough may be communicated before the rash and whoop respectively are developed. Our knowledge on this point is very limited, and facts are much needed, since its determination must materially affect the benefit to be expected from late isolation.

As to how long a disease continues to be infectious, we know very little. Dr. Squire says that, in diseases with a long incubation period, the infection generally ceases early in convalescence, whereas, in diseases with a short incubation period, infection persists far into convalescence; but of this statement, I think, we still lack the proof.

Another question which the committee is anxious to have answered is, What is the best method of disinfection to prevent the spread of disease? So far as I know, there are no facts on record to answer this question; still its solution, if disinfection be of any use, is of great importance. Chemists point out many agents which they call disinfectants, practitioners use them assiduously to the best of their ability, and still there is some doubt as to the value of any disinfectant apart from an abundance of fresh air. What we wish to know is, whether any process of disinfection can be applied so as to render the infection inert as it leaves the body of the patient. Were this possible, there would be little need for isolation. If it be impossible, why is there such faith in disinfectants?

And now, taking up the forms returned, seeing that we have not sufficient data from which to generalise, I shall, under each disease, give one or two specimens of the information we have received, and more of which is earnestly desired.

SMAlL-Pox.-A young woman, aged 19, visited, on April 16th, a village four miles distant from her own home, where there was no small-pox. She called at the house of a friend in the village. One of the daughters of this house, who was just convalescing from smallpox, tried on the visitor's bonnet. The young woman returned home, and felt nothing amiss until April 26th, when she shivered, and had other symptoms, which proved to be premonitory of small-pox.

A young woman, aged 17 , slept in the same bed with her sister until the eruption appeared on the latter; viz., until the fourth day of her illness. She was then vaccinated. The pock rose well, and she did not take small-pox.

MEASLES. - Two boys were infected at school by another boy, who became sick in the class-room without previous indisposition. The sick boy did not return to school, but stayed at home, and in due course the rash of measles showerl itself. The boy was sick on March 19th, and the two boys, who lived in different parts of the village, both became ill on. March 28th. In the house of one of these boys there were other children, two of whom, aged respectively seven months and five years, became iil on April Ioth, and a third, aged three years, on April i ith.

A boy, aged 7 , was infected by another boy, who left school on the day beforeGood Friday, I87 I, with slight cough, but had no rash till the Saturday. Exactly two weeks afterwards, the boy began with measles.

A young lady, aged 14, travelled in the same compartment of a railway carriage to Marseilles with a friend on Friday, November 8th, I873. She left her friend at Marseilles, upon whom the rash of measles was seen on Saturday or Sunday; and the young lady herself was taken ill twelve days afterward:

A boy was on a visit to a friend's house, and became ill, coughing, etc. A child in the house saw the boy for the last time on the third day of his illness, and the child's mother saw the boy on the fourth day for the last time. On the fifth day, when the rash appeared; neither the mother nor child had any communication with the invalid, but left for the seaside; exactly twelve days after leaving, the child sickened and had measles.
SCARLET FEVER. - A clerk, aged 23, received the infection from his brother, whom he neither saw nor had any communication with, direct or indirect, after $9 \mathrm{~A} . \mathrm{M}$. on January $\mathbf{I} 4 \mathrm{th}$, fourteen hours before the rash of scarlet fever appeared on his brother.

Two boys on a visit near Manchester began with scarlet fever on September 7 th. During their illness, Condy's fluid was put about the room in basins, with a view to disinfection. They went home to a solitary house near Grange on October 5 th, and one sister sickened of the same disease on October 9 th, another on October $13^{\text {th }}$, and the governess on October $15^{\text {th }}$.

Of two sisters, who were generally together, the younger became ill on November Ioth with violent sickness. They were together till the second day, when the scarlet fever rash began to show itself; and then the elder was removed to a friend's house. To disinfect, chlorine was used all through the house, and everything from the patient put into Condy's fluid and water. During desquamation, warm-baths with Condy's fluid were given, and every night the whole body was rubbed over with cod-liver oil. On December 17 th, she was carried out of the bath into another room, and put in fresh clothes. All toys, etc., were put away. Everything in the sick-room was washed with Condy's fluid in water, and the room well disinfected by competent men. She now went about the house, excepting the sick-room, with kid-gloves on, lest desquamation should continue, though none could be seen. The elder sister returned on December 2oth, and did not take the fever.

DiphtheriA.-A gentleman, who was a teacher in a school where scarlet fever existed, went home on April 3 rd with a sore-throat, which developed into well marked diphtheria. On April 9th, six days after her son's arrival, his mother, who nursed him, began with diphtheria.

A surgeon performed tracheotomy in a case of diphtheria, and with his mouth sucked some mucus up the tube from the trachea. Fourteen days afterwards, he was seized with malaise, and in two days more the exudation of diphtheria was detected.

Typhus Fever.-A practitioner, who had several cases of typhus under his care, saw no cases after a certain Monday, left home on Tuesday, and became ill on Thursday of the next week with typhus.

A practitioner began to attend a case of typhus fever with full rash on September 3 rd. Having had typhus, as he supposed, he had no fear of infection; and, amongst other things, he daily smelt the patient's breath. In ten days, he was seized with intense headache, such as he never had felt before; nevertheless, he continued working as usual until the morning of September 29th, when he attempted to rise, but was unable, and then found the spots of typhus on his skin. His wife, who slept with him till the rash was seen, and afterwards nursed him, did not take the fever. To disinfect, the windows were kept constantly open, and all discharges were immediately buried : Condy's fluid and sulphur fumigation were also used.

Typhoid Fever.-A domestic servant visited her home from June 7 th to 2 Ist, and began with typhoid fever on July I4th, having spots on July Igth. Three of her sisters were taken ill at home before her. All being confined to bed on July Ioth. A cousin who visited the house from June $17^{\text {th }}$ to 19 th, was taken ill with typhoid fever on July $13^{\text {th. }}$.

A gentleman, in the habit of visiting Rochdale every fortnight on a Wednesday, shivered as he returned the last time he was there. Typhoid was very prevalent in Rochdale, and close to where his office was. He was in the habit of having a meal when in Rochdale, and occasionally had a cup of tea in the office. There was no fever about his house, he was least at home of a large family, and he had not been, knowingly, near any fever, except in Rochdale.

HoOPING-COUGH. - A boy at school had a cough, which developed into hooping-cough ; but, ten or twelve days before he began to whoop, seven or eight of the other boys began to cough, and in due course to whoop also.

Under "Mumps", we have as yet no returns.

The following are the questions which the Committee desire to have answered.

A. Relating to Infected Persons.-I. Disease. 2. Age. 3. Sex. 4. Supposed origin of disease, whether from infection or de novo; mentioning- $a$. The length of time from supposed infection to the outbreak of the disorder ; $b$. Other possible sources of infection ; $c$. The channel through which the infection is supposed to have entered the system, e.g., by the stomach, the lungs, a wound, etc.

B. Relating to the Case from which the Infection is supposed to have arisen.-I. The time before the outbreak of the disease, or after it, at which the infection was communicated; 2 . Whether desquamation had been completed; 3. Whether disinfection had been practised; in 
what manner and to what extent, giving any facts illustrative of the value of any method of disinfection employed, as well as cases showing how soon it is safe, or unsafe, for the convalescent to mix with his fellows.

Answers may be written on ordinary note-paper, or schedules to be filled up may be had on application to me, at Monks' Hall, Eccles, Manchester.

The Committee hope that every member will endeavour to supply them with the particulars of any cases which may come under observation. Were each member to furnish us with even one case only, what a mass of facts we would possess! And, surely, the Committee's appeal will not be in vain, considering the importance of the inquiry, and the well known enthusiasm of the Associates in every undertaking which has for its object the attainment of scientific truth.

\section{CATARRH AND PYÆMIA CAUSING LARYNGEAL ABSCESS.}

\section{By THOMAS WHIPHAM, M.B., F.R.C.P.,} Assistant-Physician to St. George's Hospital.

IN the January number of the New York Medical fournal, an interesting case of abscess in the epiglottidean folds is recorded as having occurred at the Bellevue Hospital. The patient, two or three days before he was taken sick, was in excellent health, and engaged in Jefferson Market. After exposure to cold, he was attacked with swelling on the right side of the neck, and, on admission to the hospital, this was very marked on both sides, but particularly on the right. $\mathrm{He}$ suffered from dyspnœea, also due, as was supposed, to œedema of the glottis. The dyspnœa increased to such a marked extent, that laryngotomy was advised and performed. The operation relieved the dyspnœa ; but, shortly afterwards, the patient died of exhaustion. The necropsy showed that the swelling of the neck was due to a diffuse cellulitis, which had not yet undergone suppuration. In the epiglottidean folds, however, there was an abscess, which gave rise to the diagnosis of œdema glottidis. At the beginning of the present year, during the absence of Dr. Barclay for a few days, a somewhat similar case came under my care in St. George's Hospital.

A young man in domestic service, aged 25, was admitted on Feb. $3^{\text {rd, }}$ I 875 , who had five days previously been attacked with stiff neck, soon followed by headache, so severe as to compel him to take to his bed. He had then some swelling at the back of his neck, but this had almost entirely subsided at the time of his admission. The day before he came under notice, he had an attack of diarrhœa. At first, one or two spots of doubtful character on the belly led to the suspicion that it was a case of enteric fever, more especially as there was tenderness in the flanks. He was put upon bark and acid, with a small quantity of brandy, the diarrhœa of the preceding day having exhausted him considerably. On the 5th, the back of the right hand became red and painful. Similar pain and redness affected the knee and outer side of the ankle on the right side; the urine was high coloured and acid; pulse, IOO; temperature, IO4.2; bowels open once. The treatment was changed to effervescing citrate of potash with quinine every four hours, and, next day, as the pain was still severe, half a drachm of bicarbonate of potash was added to the medicine. He complained now of sore throat and slight tenderness on the left side of the neck, and the voice had become husky. Laryngoscopic examination showed a red, rounded, and opaque swelling of the mucous membrane over the left arytenoid cartilage, and, in addition, great general congestion of the larynx, but no tumefaction except as above mentioned. Small doses of antimony were ordered every two hours, of which he took about six. On the 6 th, dyspnœea accompanied by dysphagia became urgent, and the patient broke out at intervals into a profuse clammy perspiration. Various inhalations were tried, but, as they manifestly increased his distress, were discontinued. Laryngotomy was then suggested; the throat being by this time too irritable to allow any examination or treatment of the larynx itself. The patient, however, obstinately refused to submit to the operation. About 7 P.M. next day, the dyspnœa being most distressing, Mr. Harper, the house-physician, tried to obtain his consent to the insertion of a tracheal tube ; the man, however, became greatly excited, and, evidently thinking that force would be employed to compel his submission, turned quickly over on to his face in the bed, and was dead in a few seconds. The larynx was opened as soon as it was discovered that the muscles were relaxed, and artificial respiration was kept up for some time, but without effect.

Post mortem examination revealed an abscess in the soft parts on the inner surface of the left ala of the thyroid cartilage. The pus lay in contact with the cartilage, and had caused inward bulging of the ventricular band, slightly so of the vocal cord, and a certain amount of eversion of the ventricle. The normal orifice of the glottis was in consequence reduced in size. No disease of the thyroid or other cartilages existed. Microscopic examination of the swelling over the arytenoid proved the tissues to be infiltrated with a nuclear growth, which was most abundant in the neighbourhood of the blood-vessels, and immediately beneath the mucous membrane.

Cases of laryngeal abscess are at all times of great interest, and more especially so to the laryngoscopist, who may, by actual demonstration of the nature of the malady, be enabled by evacuating their contents to avert the immediate danger of suffocation, and to place the patient under conditions favourable to his recovery. But, in order to effect these results, a clear history of the formation of pus is required in addition to actual demonstration of the abscess by the laryngoscope. The above cases are placed together, with a view of showing how extremely obscure in some instances is the diagnosis of these purulent formations in the larynx.

In the first case, cold seems to have been the starting point of the disease, and this was followed by laryngitis, an event of common experience. Nothing, however, in the course of the case as reported, gave rise to a suspicion of abscess. In the second, the absence of the earlier manifestations of suppuration was remarkable; the patient had no rigors, sweatings, or any of the ordinary symptoms (save elevated temperature) of pus-formation. The most prominent feature in the case was the pain in the join's, which was at first thought to be rheumatic; but afterwards, from the brightness of the erythema, and the circumscribed area of the patches, it seemed more probable that the patient was suffering from pyæmia. Sweating certainly occurred, and profusely, towards the later periods of his life; but only after the dyspnnea became urgent. This symptom, therefure, might have been as much due to the latter cause as to the former.

A notable feature in this case is that, although after death such serious lesions were found in the larynx, there was nothing in the earlier part of the case indicating special laryngeal trouble. Swelling of the neck, doubtless, had occurred before the man's admission into hospital, but it had nearly subsided when he came under observation; and it was only after he had been under treatment for two days that special attention was directed to the throat. The voice then became husky, and the swelling over the left arytenoid was detected. This swelling, it may be well to observe, was unilateral and limited in extent; it was, therefore, not considered advisable to resort to operative interference. As the sequel proved, moreover, incision into this swelling alone would have been unproductive of good.

The throat-affection in the case which occurred at the Bellevue Hospital appears to have originated in catarrhal laryngitis, the inflammation resulting in the formation of a laryngeal abscess. Such cases are interesting by reason of their unfrequency. In my own case, the cause is less evident. Possibly it may have been due to exposure to cold; and this supposition (in the absence of any definite history) is strengthened by the occurrence of swelling and stiffness of the neck. The cold, however, will scarcely explain the disease in the larynx; and for this reason, that there was no laryngeal inflammation until the eighth day of the patient's illness. It can hardly be supposed, therefore, that laryngitis was the prime cause of the malady. The idea naturally occurred at the time of the post mortem examination, that the laryngeal abscess was but the local manifestation of general pyæmia. But as no other cause for the blood-poisoning could be discovered, this theory would seem to lack support. Nevertheless, it every now and again happens that in a case which may clinically have been considered as an undoubted case of pyæmia, the cause of the blood-puisoning, even after most diligent search, cannot be demonstrated. I am therefore disposed, seeing that the affection of the larynx did not manifest itself until so late in the patient's illness, and then as a sequela of general bloodpoisoning, to consider the abscess in this case to be of pyæmic origin.

Abscesses of the larynx, except in cases where the cartilages are diseased, are, as has been stated, rare. According to the observations of Porter, "their most usual situation is behind the broad portion of the cricoid cartilage"; and this is doubtless due to the fact, that this cartilage is more liable to be affected by caries than the thyroid epiglottis or arytenoid. "The presence of an abscess in this situation", he continues, "exercises a very decided influence in pressing upon and obstructing the rima glottidis. The approach of the suffocating symptoms is very gradual, and their progress slow ; and it is extremely difficult either to ascertain its existence or to apply a remedy." Owing to the position of the abscess in each of the recorded cases, symptoms of suffocation occurred in the natural course of events : they encroached upon and obstructed the rima glottidis. The approach of the suffocating symptoms and their progress was, however, neither so gradual 